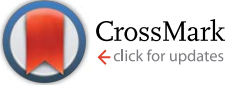

Cite this: RSC Adv., 2017, 7, 15971

\title{
Enhanced anticancer effect of copper-loaded chitosan nanoparticles against osteosarcoma
}

\begin{abstract}
Jin-wei $\mathrm{Ai}^{\text {a }}{ }^{\text {Wen Liao*b }}$ and Zhi-Long Ren ${ }^{\mathrm{c}}$
In this study, copper-loaded chitosan nanoparticles were prepared for the effective treatment of osteosarcoma. The particle size of the copper-loaded chitosan nanoparticles (CuCNPs) is in the range required for drug delivery applications and cancer targeting $(<200 \mathrm{~nm})$. The spherical shape of the nanoparticles was observed via TEM and AFM. CUCNPs exhibited a significantly higher anticancer effect compared to free copper sulphate $\left(\mathrm{CuSO}_{4}\right)$. The substantial anticancer effect of CuCNPs might be due to the better internalization of the NP system compared to that of $\mathrm{CuSO}_{4}$. CuCNPs exhibited a remarkable anticancer effect on cancer cells, where much of the cells were dead and few had typical apoptotic body formations. Our results clearly reveal that the superior anticancer effect of CuCNPs is attributed to the generation of a higher mitochondrial ROS level compared to that of the control. Caspase 3/7 activity showed that CuCNPs exhibit 6-fold and 2-fold higher apoptotic activities than the control and $\mathrm{CuSO}_{4}$ groups, respectively. Based on these result, it can be speculated that the higher ROS generation capacity of CuCNPs result in higher caspase 3/7 activity, which is further confirmed by the higher expression of caspase-3. Overall, the anticancer effect of copper could be enhanced by delivering it in biocompatible chitosan nanoparticles for effective therapy in osteosarcoma.
\end{abstract}

Received 29th August 2016

Accepted 20th December 2016

DOI: $10.1039 / c 6 r a 21648 j$

rsc.li/rsc-advances biocompatibility, and antimicrobial characteristics. In addition, chitosan could be cross-linked with tripolyphosphate (TPP) and form stable nanoparticles that are used for the delivery of several anticancer drugs and oligonucleotides. ${ }^{\mathbf{1 0 , 1 1}}$ It has been reported that chitosan NPs could target cancer cells by preferentially accumulating in the tumor tissues by virtue of the enhanced permeation and retention (EPR) effect and overcome the multidrug resistance induced by p-glycoprotein. ${ }^{\mathbf{1 2}}$ Chitosan has been reported to form several complexes, such as chitosansilver, chitosan-zinc, and chitosan-manganese, and is active against several bacterial strains, such as Escherichia coli, Salmonella choleraesuis, and Staphylococcus aureus, in vitro. ${ }^{\mathbf{1 3 - 1 5}}$ The superior cytotoxic activity of the chitosan-metal complex is mainly attributed to the high affinity of metal ions towards the cell membrane.

In the present study, we employed copper as a new metal ion and complexed it with chitosan. $\mathrm{Cu}$ (II) ions could be effectively adsorbed on the chitosan surface at $\mathrm{pH} 6 .{ }^{\mathbf{1 6}}$ Recently, the excellent antibacterial property of copper against gram +ve and -ve bacteria and cytotoxicity potential against multiple cancer cells have been reported. ${ }^{17,18}$ Although copper has been shown to possess anticancer activity in certain cancer cells, there is no study on its efficacy in osteosarcoma.

The main aim of the present study was to investigate the anticancer property of copper or a copper-loaded NP system in osteosarcoma cells (MG-63). For this purpose, copper was surface adsorbed on a preformed chitosan NP. The high affinity of the $-\mathrm{NH}_{2}$ or $-\mathrm{OH}$ groups of chitosan NP towards copper
${ }^{a}$ Department of Orthopedics, Henan University of Chinese Medicine, 450002, China ${ }^{b}$ Department II of Orthopedics, Wuhan Third Hospital, No.216, Guangshan Avenue, Hongshan District, Wuhan, 430074 China.E-mail: liaowen245@hotmail.com; Fax: +86-27-87801006; Tel: +86-27-87801006

${ }^{c}$ Department I of Orthopedics, The First AHospital of Yulin, 719000, China 
increases the stability of the metal ion. Moreover, it was expected that copper coated on the NP system will have a higher cellular uptake compared to the free metal itself. We first differentiated the size difference between bare and coppercoated chitosan NPs. Subsequently, the morphology of the bare and copper-coated chitosan NPs (CuCNPs) was evaluated via TEM and AFM. Importantly, the anticancer effect of the blank NPs, $\mathrm{CuSO}_{4}$, and CuCNPs was studied on the MG-63 osteosarcoma cells using the MTT assay. Especially, reactive oxygen species (ROS) generation after treatment with the individual formulation was investigated via confocal microscopy. Finally, the apoptosis activity of the individual formulations was studied by caspase $3 / 7$ activity and an immunoblotting experiment was performed to further confirm the apoptosis effect of the formulations.

\section{Results and discussion}

To develop a new approach to treat osteosarcoma while decreasing the associated side effects of chemotherapy, in the present study, we report copper-coated chitosan NPs (CuCNPs). The CuCNPs were characterized for their anticancer efficacy against osteosarcoma. For this purpose, copper was surface adsorbed on a preformed chitosan NP. The high affinity of the $-\mathrm{NH}_{2}$ or $-\mathrm{OH}$ groups of chitosan NP towards copper increases the stability of the metal ion (Fig. 1).

The size distribution and surface charge of the NP system are vital parameters for successful systemic applications. As shown in Fig. 2, the bare chitosan NP (CNP) has a mean particle size of $\sim 110 \mathrm{~nm}$ with a narrow polydispersity index (PDI $\sim 0.15$ ). As expected, the mean particle size of CuCNP increased to $\sim 190 \mathrm{~nm}$ upon the loading of copper, indicating a firm loading of metal on the surface of the chitosan NP (Fig. 2). The high affinity of the
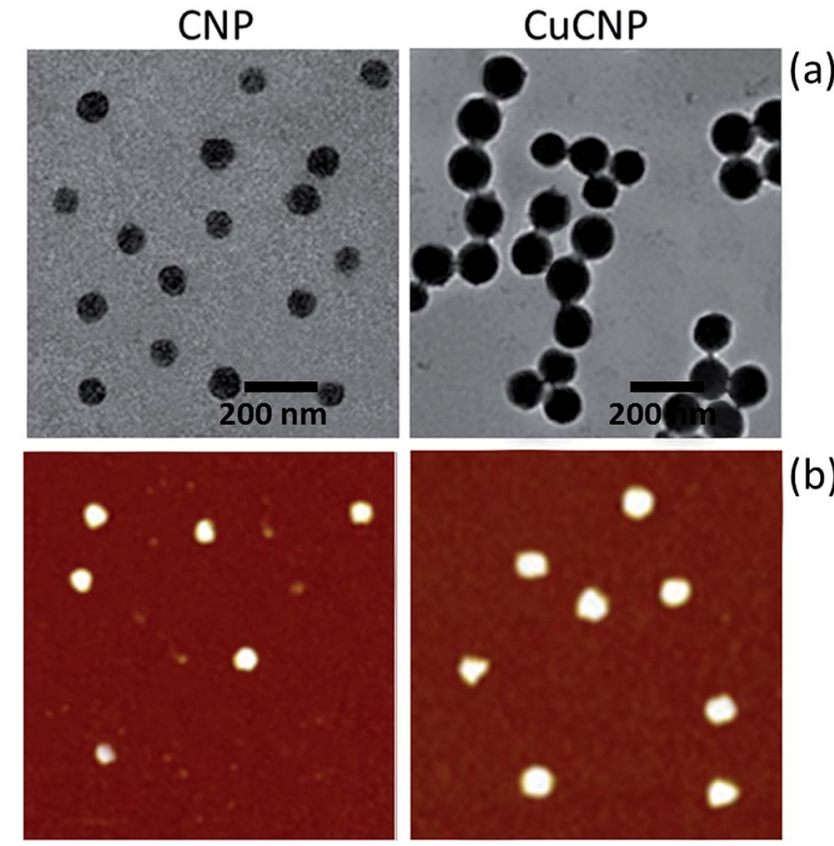

Fig. 3 (a) Particle shape analysis of CNP and CuCNP using transmission electron microscopy (TEM) and atomic force microscopy (AFM)

chitosan NP towards metal ions is responsible for this high loading. ${ }^{19}$ However, the overall size of the nanoparticles was $<200 \mathrm{~nm}$, which indicates their suitability for cancer targeting applications. It has been reported that a size less than $<200 \mathrm{~nm}$ will gain access into tumor tissues by virtue of the EPR effect. ${ }^{20}$ Furthermore, the zeta potential of both nanoparticles was determined. The zeta potential of CNP was $+28.5 \pm 2.16 \mathrm{mV}$,

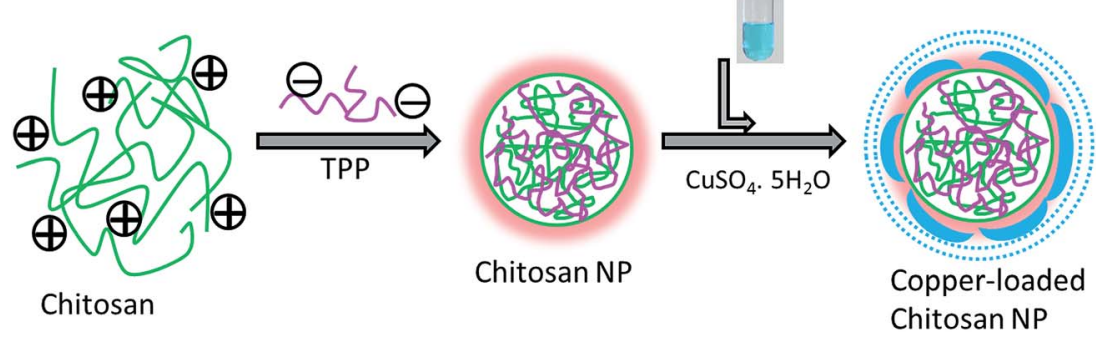

Fig. 1 Schematic of the preparation of copper-loaded chitosan nanoparticles using TPP as a cross-linking agent.
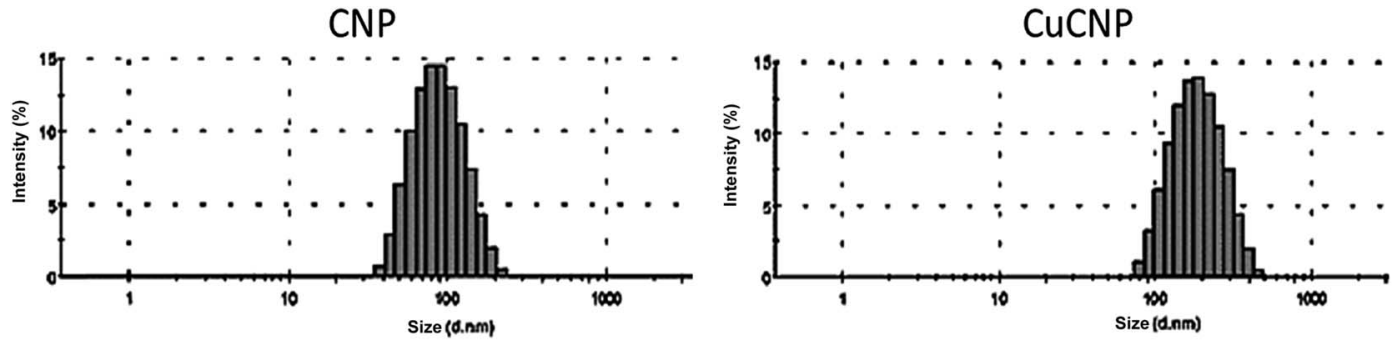

Fig. 2 Particle size distribution of CNP and CuCNP using the dynamic light scattering (DLS) method. 


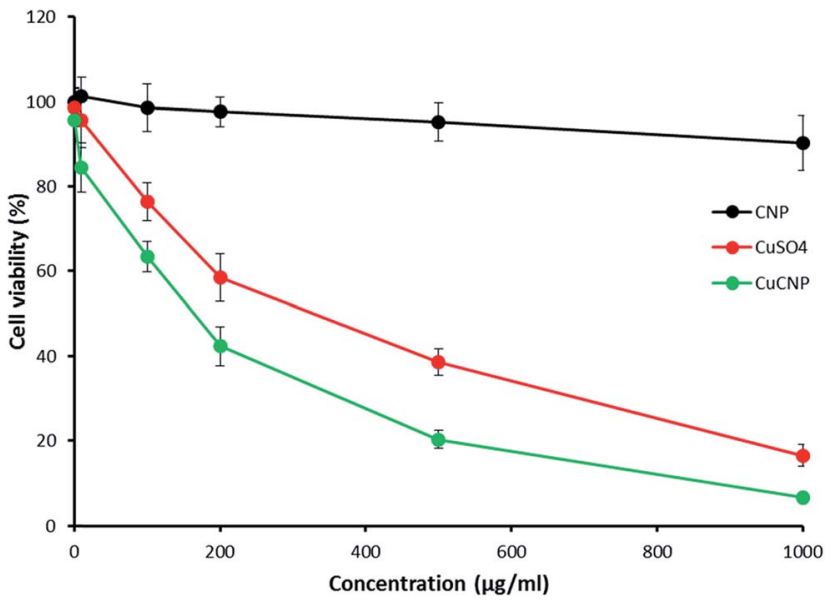

Fig. 4 Cytotoxicity analysis of the MG-63 osteosarcoma cancer cells after treatment with blank NP, $\mathrm{CuSO}_{4}$, and CuCNP. The cytotoxicity analysis was performed via the MTT assay.

which increased to $+41.4 \pm 1.65 \mathrm{mV}$ for CuCNP, implying the successful loading of the metal ion on the NP surface. This increase in the surface charge was attributed to the positive charge of copper ions. It has been reported that copper ions at first adsorb on the surface of NP, whereas they diffuse into the pores or cavity of the NP with time and chelate on the internal surface of the NP. The entrapment efficiency of $\mathrm{Cu}$ was found to be $82.56 \pm 3.5 \%$ with a loading capacity of $\sim 16 \% \mathrm{wt} / \mathrm{wt}$.

\section{Morphological analysis}

The morphology of the NP was first studied via TEM. It can be seen that CNP and CuCNP were typically spherical in nature and uniformly distributed on the grid surface. Note that the copper loading did not change the spherical morphology of the nanoparticles (Fig. 3a). Consistent with the DLS observation, CuCNPs showed a larger particle size compared to that of the bare NP. A discrepancy was observed in the particle size observed from DLS and TEM. For example, DLS showed a mean particle size of $\sim 190 \mathrm{~nm}$ for CuCNPs, whereas TEM showed a mean particle size of $\sim 150 \mathrm{~nm}$. This decrease in the particle size was due to the presence of particles in the dried state, whereas DLS is observed in the hydrodynamic state. The particle size was further confirmed by AFM (Fig. 3b). The particles were uniformly spread on the mica surface and clearly spherical in nature. As observed in TEM, the particle size of the CuCNPs was relatively larger than that of the CNPs.

\section{In vitro anticancer activity}

The in vitro anticancer efficacy of the bare $\mathrm{CNPs}, \mathrm{CuSO}_{4}$, and CuCNPs was studied in MG-63 osteosarcoma cancer cells using the MTT assay. As observed, the bare CNPs had no effect on the cell proliferation of MG63 cells and the cell viability remained more than $90 \%$ even at the highest concentration tested. These results further suggest the excellent biocompatibility profile of chitosan NP, implying their suitability for systemic or tumor targeting applications (Fig. 4). In contrast, $\mathrm{CuSO}_{4}$ and CuCNPs exhibit a typical concentration-dependent cytotoxic effect in cancer cells. Specifically, CuCNPs exhibited a significantly higher anticancer effect compared to free $\mathrm{CuSO}_{4}$. The substantial anticancer effect of the CuCNPs might be due to the better internalization of the NP system compared to that of $\mathrm{CuSO}_{4}$. It has been earlier reported that chitosan NP efficiently internalize cancer cells owing to the interaction of the positively charged NP with the negatively charged surface of the cell membrane. It has been repeatedly demonstrated that NP escapes the lysosomal compartments and enters the cytoplasmic region. The NP then remains in the cytoplasm and releases the encapsulated compound in a sustained manner, resulting in an enhanced therapeutic effect compared to that of the free drug. It has been already reported that metal NP exhibits an anticancer effect by causing oxidative stress, apoptosis, and inflammation to endothelial cells. ${ }^{21,22}$ The anticancer effect of the individual formulations was further evaluated via morphological examinations. As observed, the cells treated with blank NP maintained their typical morphology and remained similar to the untreated cells, which further confirms their biocompatible nature. However, $\mathrm{CuSO}_{4}$ reduced the cell number on the cover slip and the cells were very much distorted and irregular in shape, which indicate the cell death effect.

Additionally, we performed individual experiments on the CHO cells and MC3T3-E1 preosteoblast cells. The results reveal that copper sulphate and copper sulphate nanoparticles are
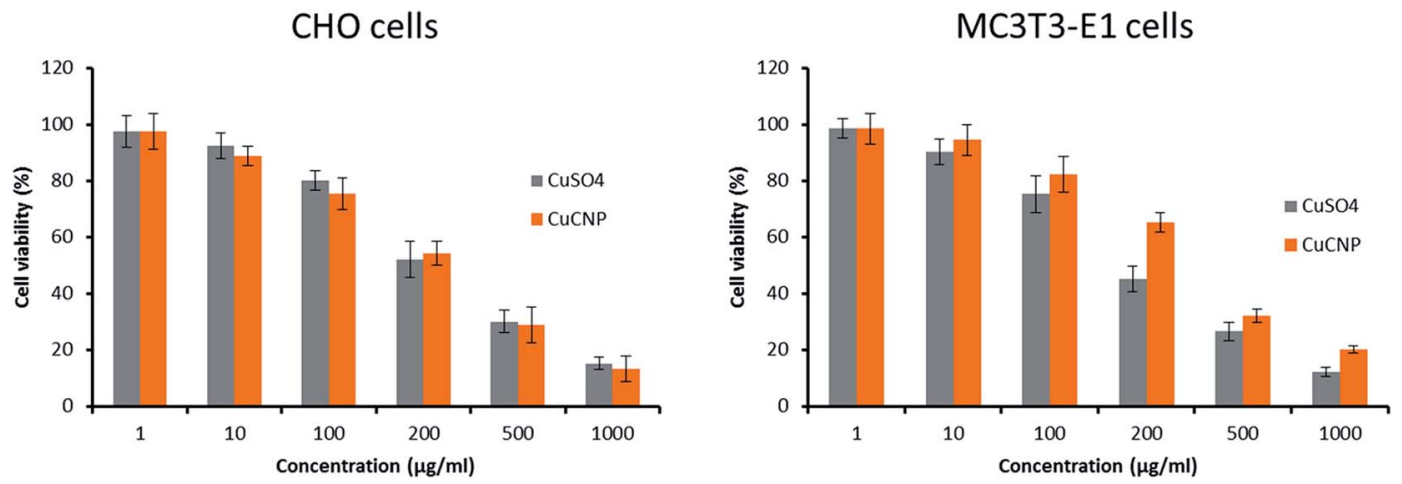

Fig. 5 Cytotoxicity analysis of $\mathrm{CHO}$ cells and MC3T3-E1 normal cells after treatment with $\mathrm{CuSO}_{4}$ and CuCNP. The cytotoxicity analysis was performed via the MTT assay. 

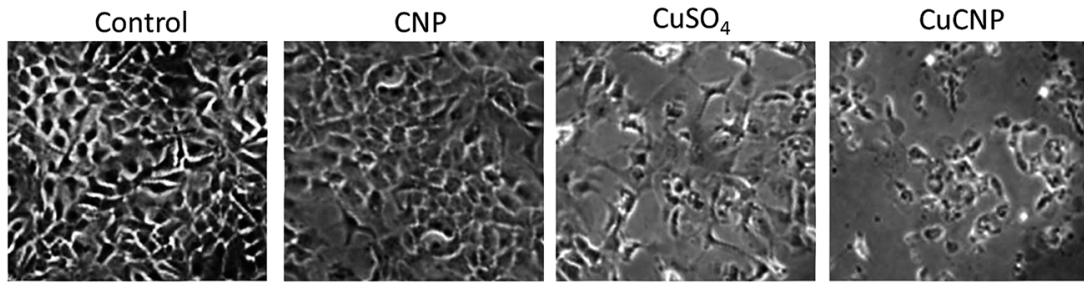

Fig. 6 Morphological analysis of MG-63 osteosarcoma cancer cells after treatment with blank NP, CuSO ${ }_{4}$ and CuCNP.

toxic to normal healthy cells in a concentration-dependent manner. Earlier, we showed that CuCNP shows a typical concentration-dependent toxicity in osteosarcoma tumor cells. Their cytotoxic action on cancer cells is different from their action on normal cells. Under in vitro conditions, $100 \%$ of the free copper(II) or NP internalize the healthy cells and therefore toxicity is observed. Based on the studies and nano-sized nature of these particles, we expected that the biocompatible chitosanloaded copper nanoparticles will be preferentially accumulated in cancer cells via the EPR effect and will have limited interaction with healthy cells. Therefore, it can be expected that although CuCNP is toxic to healthy cells under in vitro conditions, it will be much safer under in vivo conditions. A study in this perspective is the subject of our ongoing research.

Importantly, CuCNP exhibited a remarkable effect on cancer cells with maximum damage to their structural integrity and properties (Fig. 5). Much of the cells were dead and fewer in number with typical apoptotic body formations, indicating their superior anticancer effect compared to that of free copper ions.

\section{In vitro reactive oxygen species (ROS) analysis}

Metal NPs are known to produce an anticancer effect by generating ROS in the intracellular environment that cause oxidative damage, DNA fragmentation, and eventually cell death. The ROS have been considered as a prime hallmark of cellular damage and cellular apoptosis..$^{23}$ Moreover, an exposure to metal NP results in an increase in the intracellular ROS, which cause damage to the mitochondria and cellular organelles and cause cell death. In the present study, we employed DCFH-DA to track the ROS generation in the cancer cells after treatment with the respective formulations. DCFH-DA diffuses into viable cancer cells via plasma membrane, hydrolyzes to $\mathrm{DCFH}$, and further oxidation converts it into the fluorescent form. Our results clearly reveal the superior anticancer effect of CuCNP, which generates a higher mitochondrial ROS level compared to the control (Fig. 6). A similar concentration of free $\mathrm{CuSO}_{4}$ did not result in higher ROS generation. Similar results were obtained via RFU analysis using a luminometer. These results, therefore, clearly demonstrate the superior ROS generation capacity of the NP-based copper that results in a higher anticancer effect.

\section{In vitro apoptosis analysis and immunoblot assay}

To further confirm the anticancer effect of the individual formulations, a caspase 3/7 activity-based apoptosis assay was carried out. Caspases are a family of cysteine proteases that play
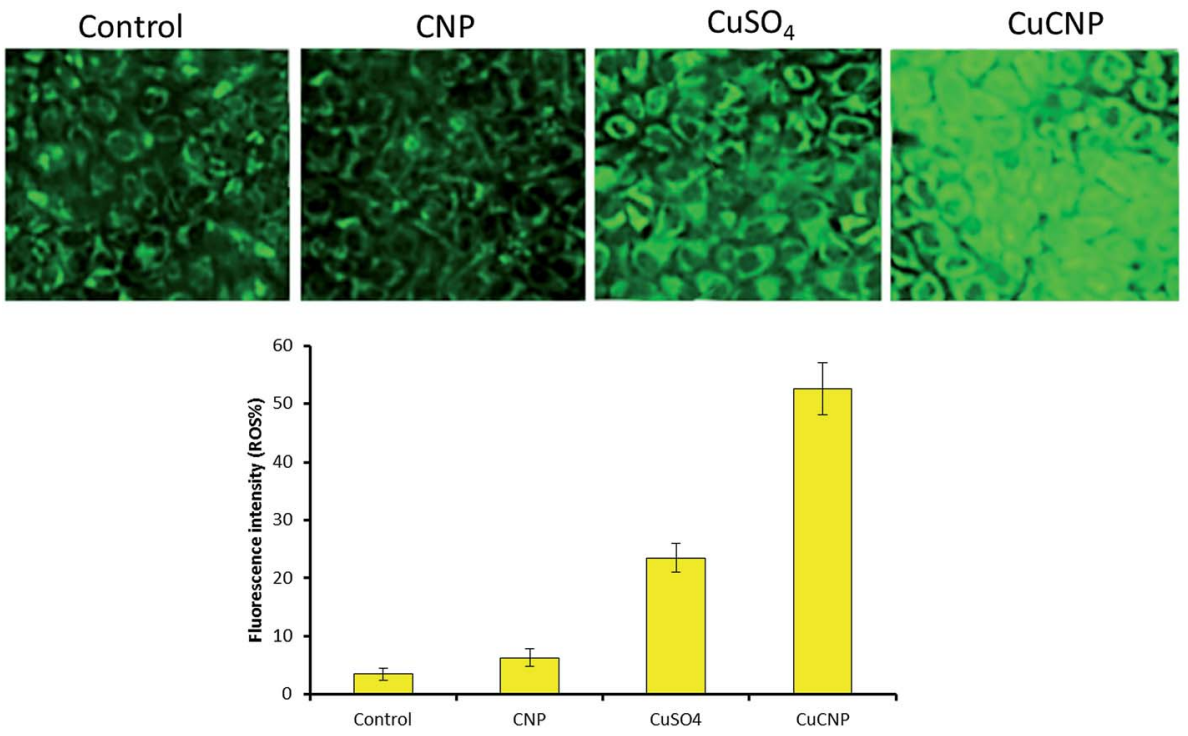

Fig. 7 The generation of reactive oxygen species (ROS) in the intracellular environment of cancer cells (MG63) after treatment with blank NP, $\mathrm{CuSO}_{4}$, and CuCNP. The ROS analysis was performed by measuring the fluorescence intensity of dichlorofluorescein (DCF), which is formed via the reduction of 2,7-dichloro-fluorescein-diacetate (DCFH-DA). 
(a)

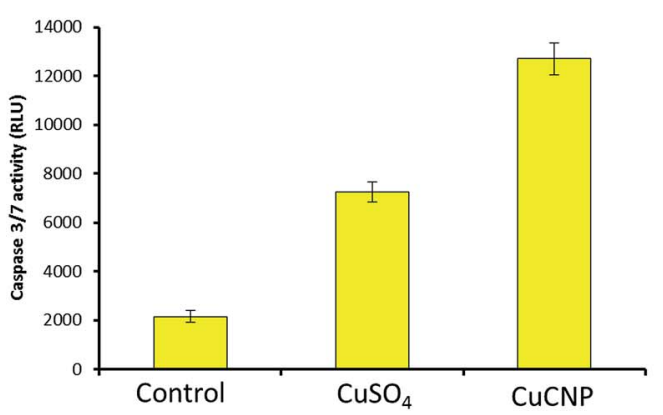

(b)

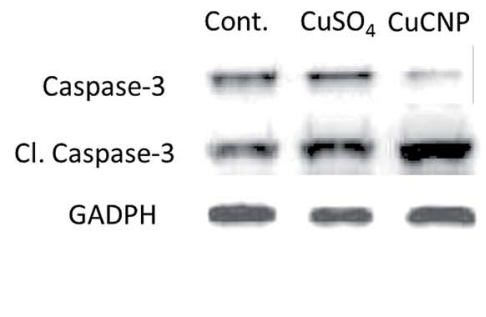

Fig. 8 (a) Caspase activity measured using the commercial Caspase-Glo 3/7 Assay Kit (Promega) with the help of a microplate reader luminometer after treatment with $\mathrm{CuSO}_{4}$ and $\mathrm{CuCNP}$ and (b) immunoblot analysis of cancer cells after treatment with $\mathrm{CuSO}_{4}$ and $\mathrm{CuCNP}$.

essential roles in mediating apoptosis. It is known that the mitochondrion plays an important role in maintaining the structural integrity of cells and directs the death signal pathways. Moreover, it controls several key signaling pathways responsible for the apoptosis cascade and release of caspase activators. The results show that CuCNP exhibits 6-fold higher apoptosis activity and 2-fold higher activity than the control and $\mathrm{CuSO}_{4}$ groups, respectively (Fig. 7a). This higher caspase activity is consistent with the previous studies that reported a superior effect for nanoparticle-based copper. Based on this result, it can be speculated that the higher ROS generation capacity of CuCNP results in the higher caspase $3 / 7$ activity. To further validate this, an immunoblot analysis was performed to check the expression levels of the original as well as the cleaved caspase-3 upon treatment with $\mathrm{CuSO}_{4}$ and CuCNP, respectively. As expected, cleavage of caspase-3 was higher in the formulation-treated cells, which confirms its higher anticancer effect (Fig. 7b). Results further confirm that the apoptotic effect of copper was initiated from the activation of caspases. Overall, the increased ROS levels and changes in the mitochondrial potentials might be the reason behind the higher apoptosis for CuCNP (Fig. 8).

\section{Conclusion}

In summary, copper-loaded chitosan nanoparticles were successfully prepared for the effective treatment of osteosarcoma. The particle size of the CuCNP was in the range required for drug delivery applications and cancer targeting $(<200 \mathrm{~nm})$. The spherical shape of the nanoparticles was observed via TEM and AFM. CuCNP exhibited a significantly higher anticancer effect compared to free $\mathrm{CuSO}_{4}$. The substantial anticancer effect of CuCNP might be due to the better internalization of the NP system compared to that of $\mathrm{CuSO}_{4}$. CuCNP exhibited a remarkable anticancer effect on cancer cells where much of the cells were dead and few had typical apoptotic body formations. Our results clearly reveal that the superior anticancer effect of CuCNP is attributed to the generation of a higher mitochondrial ROS level compared to that of the control. The caspase $3 / 7$ activity shows that CuCNP exhibits 6-fold and 2-fold higher apoptotic activities than the control and $\mathrm{CuSO}_{4}$ groups, respectively. Based on these results, it can be speculated that the higher ROS generation capacity of CuCNP results in the higher caspase $3 / 7$ activity, which is further confirmed by the higher expression of caspase-3. Overall, the anticancer effect of copper could be enhanced by delivering it in biocompatible chitosan nanoparticles for effective therapy in osteosarcoma.

\section{Materials and methods}

\section{Materials}

Low-molecular weight chitosan (85\% deacetylated), $\mathrm{CuSO}_{4}$, and TPP were purchased from Sigma-Aldrich, China. All other chemicals were of reagent grade and used without further purifications.

\section{Preparation of copper-loaded chitosan nanoparticles}

The chitosan NPs were prepared via the ionic gelation method. Briefly, $50 \mathrm{mg}$ of chitosan was dissolved in $0.5 \%$ of acetic acid solution $(8 \mathrm{~mL})$ and stirred for $4 \mathrm{~h}$. The chitosan NPs were then prepared by the addition of a TPP solution $(5 \mathrm{mg})$ in a dropwise manner. The solution was stirred for $2 \mathrm{~h}$, and then the NPs were separated by centrifugation at $14000 \mathrm{rpm}$ for $10 \mathrm{~min}$. The NPs were freeze-dried and the mass of the NPs was carefully weighed. Then, copper-loaded chitosan NPs (CuCNP) were prepared by the addition of 1 part of $\mathrm{CuSO}_{4}$ to 5 parts chitosan NPs and the mixture was stirred for $15 \mathrm{~min}$ at $500 \mathrm{rpm}$ per min. The CuCNPs were then stored under $8{ }^{\circ} \mathrm{C}$ until further use. In the present study, we employed low-molecular weight chitosan ( $\sim 50000 \mathrm{Da})$ with an average viscosity of $\sim 100 \mathrm{cP}$. The $\mathrm{Cu}$ content was determined via ICP-MS (Sector Field SF-ICP-MS Element 2 from Thermo Finnigan, detection limit: 0.004 $\left.\mu \mathrm{g} \mathrm{L^{-1 }}\right)$.

\section{Particle size and surface charge analysis}

The particle size and surface charge of the NPs were evaluated using the dynamic light scattering (DLS) technique. A Zetasizer Nano-ZS (Malvern Instruments, Malvern, UK) was used to measure the size and surface charge characteristics. The sample $(100 \mu \mathrm{L})$ was diluted to $1000 \mu \mathrm{L}$ and the experiment was performed in triplicate. 


\section{Morphological analysis}

The morphology of the nanoparticles was first evaluated by transmission electron microscopy (TEM). For this purpose, a drop of the sample was placed on a TEM grid (300 mesh; Ted Pella, CA, USA) and excess liquid was wiped off. The sample was then counter-stained with a negative staining agent and airdried. The particles were observed under a Tecnai 12 microscope (FEI, Hillsboro, OR, USA) equipped with a CCD camera, Gaten, Inc (Pleasanton, CA, USA) at $100 \mathrm{keV}$. For the AFM analysis, samples were placed on a mica surface and air-dried. AFM images were observed using a Nanoscope IIIa instrument under tapping mode.

\section{Cell culture and cytotoxicity assay}

The human osteosarcoma cell line MG-63 was purchased from the Health Science Research Resources Bank (Osaka, Japan). The cells were maintained in an RPMI 1640 medium supplemented with $10 \%$ fetal bovine serum at $37{ }^{\circ} \mathrm{C}$ in $5 \% \mathrm{CO}_{2}$ in a humidified atmosphere. Chinese hamster ovary $(\mathrm{CHO})$ cells were purchased from the China Center for Type Culture Collection, Wuhan, China. The CHO cells were cultured in an RPMI medium. RPMI media, FBS, and antibiotic mixtures were purchased from Sigma-Aldrich, China. The mouse osteoblastic cell line MC3T3-E1 was obtained from the American Type Culture Collection (ATCC, Rockville, MD). The MC3T3-E1 cells were cultured in $\alpha$-MEM (Gibco BRL, Gaithersburg, MD).

The MG-63 cancer cells were grown in an RPMI medium supplemented with $10 \%$ FBS and 1\% antibiotic mixture. The cells were maintained in a humidified incubator with $5 \% \mathrm{CO}_{2}$ at $37^{\circ} \mathrm{C}$. For the MTT assay, $1 \times 10^{4}$ cells were seeded in each well of a 96-well plate and incubated overnight. The next day, the medium was removed and replaced with fresh medium containing blank NP, $\mathrm{CuSO}_{4}$, and CuCNP and incubated for $24 \mathrm{~h}$. The medium was carefully removed and washed two times with PBS. The cells were treated with MTT solution $(10 \mu \mathrm{L}, 5 \mathrm{mg}$ $\mathrm{mL}^{-1}$ ) and further incubated for $4 \mathrm{~h}$. Then, $100 \mu \mathrm{L}$ DMSO was added and after $15 \mathrm{~min}$, the absorbance was read using an M200 Pro reader at $570 \mathrm{~nm}$.

The morphology of the cancer cells after treatment with the individual formulation was observed using an optical microscope.

\section{Reactive oxygen species (ROS) analysis}

The ROS analysis was performed by measuring the fluorescence intensity of dichlorofluorescein (DCF), which was formed via the reduction of 2,7-dichloro-fluorescein-diacetate (DCFH-DA) in the intracellular environment. Briefly, $2.5 \times 10^{5}$ cells were seeded in each well of a 12-well plate and incubated overnight. The cells were then treated with blank NP, $\mathrm{CuSO}_{4}$, and CuCNP and incubated for $24 \mathrm{~h}$. The cells were then incubated with 10 $\mu \mathrm{L}$ of DCFH-DA for $30 \mathrm{~min}$. The cells were then washed thrice with PBS and the images were obtained using a confocal microscope. A fluorospectrometer was also used to calculate the intracellular ROS generation and the values are presented as percentages.

\section{Apoptotic analysis by caspase $3 / 7$ activity}

$1 \times 10^{4}$ cells were seeded in each well of a 96-well plate and incubated overnight. The next day, the medium was removed and replaced with fresh medium containing blank $\mathrm{NP}, \mathrm{CuSO}_{4}$, and CuCNP and incubated for $24 \mathrm{~h}$. Caspase-Glo (3/7) reagent (Promega) $(100 \mu \mathrm{L})$ was added to the 96-well plate and incubated for $30 \mathrm{~min}$. The reading was observed from a luminometer and the values are presented as relative luminescence units (RLU).

\section{Western blot analysis}

$0.5 \times 10^{6}$ cells were seeded in each well of a 6 -well plate and incubated overnight. The next day, the medium was removed and replaced with fresh medium containing $\mathrm{CuSO}_{4}$, and CuCNP and incubated for $24 \mathrm{~h}$. The cells were washed twice and collected by trypsinization. The cells were again washed and lysed using lysis buffer (50 mM Tris- $\mathrm{HCl}$ pH 7.4, $137 \mathrm{mM} \mathrm{NaCl,}$ $10 \%$ glycerol, $100 \mathrm{mM}$ sodium vanadate, $1 \mathrm{mM}$ PMSF, $10 \mathrm{mg}$ $\mathrm{mL}^{-1}$ aprotinin, $10 \mathrm{mg} \mathrm{mL}^{-1}$ leupeptin, $1 \% \mathrm{NP}-40$, and $5 \mathrm{mM}$ cocktail). The lysed cells were centrifuged and the supernatant was collected and determined for protein level using the bicinchoninic acid assay (BCA) method. An equal amount of proteins $(35 \mu \mathrm{g})$ was loaded on $10 \%$ polyacrylamide gel and the proteins were transferred to PVDF membrane. The membrane was blocked using 5\% non-fat dry milk and washed with TBST. The membranes were incubated with primary antibodies for original caspase- 3 and cleaved caspase- 3 and left overnight. The primary and secondary antibodies were purchased from Santa Criz Biotechnology, CA, 95060, USA. The membranes were washed and incubated with secondary antibody for $1 \mathrm{~h}$. Then, Xray autoradiography was performed and the grey scale images were analyzed.

\section{Statistical analysis}

The data are presented as the mean \pm SD obtained from three or six individual experiments. The values were analysed by oneway ANOVA (analysis of variance). All statistical analyses were performed using the SPSS software (version 17.0, SPSS, USA).

\section{Acknowledgements}

The study was supported from the funding grant of Henan University of Chinese Medicine, China.

\section{Notes and references}

1 A. Longhi, C. Errani, M. De Paolis, M. Mercuri and G. Bacci, Cancer Treat. Rev., 2006, 32, 423-436.

2 A. J. Chou and R. Gorlick, Expert Rev. Anticancer Ther., 2006, 6, 1075-1085.

3 N. De Saint Aubain Somerhausen and C. D. Fletcher, Eur. J. Surg. Oncol., 1999, 25, 215-220.

4 G. Boos and H. Stopper, Toxicol. Lett., 2000, 116, 7-16.

5 V. V. Mody, R. Siwale, A. Singh and H. R. Mody, J. Pharm. BioAllied Sci., 2010, 2, 282. 
6 A. M. Schrand, M. F. Rahman, S. M. Hussain, J. J. Schlager, D. A. Smith and A. F. Syed, Wiley Interdiscip. Rev.: Nanomed. Nanobiotechnol., 2010, 2, 544-568.

7 H. A. Jeng and J. Swanson, J. Environ. Sci. Health, Part A: Toxic/Hazard. Subst. Environ. Eng., 2006, 41, 2699-2711.

8 S. Bokern, J. Getze, S. Agarwal and A. Greiner, Polymer, 2011, 52, 912-920.

9 M. Valodkar, P. S. Rathore, R. N. Jadeja, M. Thounaojam, R. V. Devkar and S. Thakore, J. Hazard. Mater., 2012, 201, 244-249.

10 T. Ramasamy, T. H. Tran, H. J. Cho, J. H. Kim, Y. I. Kim, J. Y. Jeon and H. G. Choi, Pharm. Res., 2014, 31, 1302-1314.

11 T. Ramasamy, T. H. Tran, J. Y. Choi, et al., Carbohydr. Polym., 2014, 102, 653-661.

12 T. Ramasamy, H. B. Ruttala, N. Chitrapriya, et al., Acta Biomater., 2017, 48, 131-143.

13 S. P. Chen, G. Z. Wu and H. Y. Zeng, Carbohydr. Polym., 2005, 60, 33-38.

14 X. H. Wang, Y. M. Du and H. Liu, Carbohydr. Polym., 2004, 56, 21-26.
15 M. J. Domek, M. W. LeChevallier and G. A. McFeters, Appl. Environ. Microbiol., 1984, 48, 289-293.

16 F. Rispoli, A. Angelov, D. Badia, A. Kumar, S. Seal and V. Shah, J. Hazard. Mater., 2010, 180, 212-216.

17 W. L. Du, Y. L. Xu, Z. R. Xu and C. L. Fan, Nanotechnology, 2008, 19, 085707.

18 K. Kasemets, A. Ivask, H.-C. Dubourguier and A. Kahru, Toxicol. In Vitro, 2009, 23, 1116-1122.

19 A. S. Choplin and F. O. Quignard, Coord. Chem. Rev., 1998, 178, 1679-1702.

20 T. Ramasamy, Z. S. Haidar, T. H. Tran, J. Y. Choi, J. H. Jeong, B. S. Shin and H. G. Choi, Acta Biomater., 2015, 10, 51165127.

$21 \mathrm{~J}$. H. Niazi and M. B. Gu, Atmospheric and Biological Environmental Monitoring, 2009, pp. 193-206.

22 O. Bondarenko, K. Juganson, A. Ivask, K. Kasemets, M. Mortimer and A. Kahru, Arch. Toxicol., 2013, 87, 11811200.

23 M. Murphy, Biochem. J., 2009, 417, 1-13. 